\title{
Impacto da pandemia de Covid-19 no atendimento ao paciente com hanseníase: estudo avaliativo sob a ótica do profissional de saúde
}

Impact of the Covid-19 pandemic on the care of patients with leprosy: an evaluative study from the perspective of the health professional

Impacto de la pandemia de Covid-19 en el cuidado de pacientes con lepra: un estudio evaluativo desde la perspectiva del profesional de la salud

\begin{abstract}
Resumo
Este estudo objetivou analisar o impacto da pandemia de Covid-19 no atendimento em hanseníase sob a ótica do profissional de saúde. Trata-se de um estudo de avaliação de serviços, transversal, descritivo, através de questionário impresso, destinado a profissionais de saúde médicos, enfermeiros e fisioterapeutas. O estudo foi realizado com 17 profissionais de saúde, sendo 7 enfermeiros, 7 médicos e 3 fisioterapeutas pertencentes a 7 unidades de saúde da atenção primária em um município paraense. Observou-se que apenas $24 \%$ dos profissionais entrevistados passaram por remanejamento de setor, $82 \%$ dos profissionais afirmaram que houve mudanças no perfil de atendimento das unidades de saúde, houve necessidade de reagendamentos de atendimentos para os pacientes, $88 \%$ dos profissionais passaram por rodízio de atividades, $47 \%$ afirmaram que houve falta de medicamentos para o tratamento da hanseníase nas unidades em que eles atuam, 53\% afirmaram que não houve falta de materiais para o cuidado do paciente com hanseníase, houve dificuldades no agendamento de exames laboratoriais, na avaliação de contatos domiciliares e sociais e na referência e contrarreferência dos pacientes. A partir destes dados conclui-se que a pandemia de Covid-19 criou dificuldades para o atendimento e tratamento do paciente com hanseníase nas unidades de saúde da atenção primária em saúde participantes, gerando atrasos em agendamentos, remarcações de consultas, prorrogações de tratamento devido à falta de medicação, dificuldades na avaliação de contatos e busca ativa de novos casos, contribuindo para gerar um impacto negativo no programa e consequentemente no sistema de saúde.
\end{abstract}

Palavras-chave: Hanseníase; Covid-19; Pandemia; Profissionais de saúde.

\begin{abstract}
This study aimed to analyze the impact of the Covid-19 pandemic on leprosy care from the perspective of health professionals. This is a descriptive, cross-sectional, service evaluation study using a printed questionnaire, aimed at medical health professionals, nurses and physiotherapists. The study was carried out with 17 health professionals, 7 nurses, 7 physicians and 3 physiotherapists belonging to 7 primary care health units in a municipality in Pará. It was observed that only $24 \%$ of the professionals interviewed underwent sector relocation, $82 \%$ of professionals said that there were changes in the service profile of the health units, there was a need to reschedule care for patients, $88 \%$ of professionals underwent rotation of activities, $47 \%$ said there was a lack of medication for the treatment of leprosy in the units where they work, 53\% said there was no lack of materials for the care of leprosy patients, there were
\end{abstract}


difficulties in scheduling laboratory tests, in the evaluation of home and social contacts and in the referral and counter-referral of patients. From these data, it is concluded that the Covid-19 pandemic created difficulties for the care and treatment of patients with leprosy in participating primary health care units, causing delays in appointments, rescheduling of appointments, extensions of treatment due to lack of medication, difficulties in evaluating contacts and active search for new cases, contributing to generate a negative impact on the program and consequently on the health system.

Keywords: Leprosy; Covid-19; Pandemic; Health professionals.

\section{Resumen}

Este estudio tuvo como objetivo analizar el impacto de la pandemia Covid-19 en la atención de la lepra desde la perspectiva de los profesionales de la salud. Se trata de un estudio de evaluación de servicios, descriptivo, transversal, mediante cuestionario impreso, dirigido a profesionales médicos, enfermeros y fisioterapeutas. El estudio se realizó con 17 profesionales de la salud, 7 enfermeras, 7 médicos y 3 fisioterapeutas pertenecientes a 7 unidades de atención primaria de salud de un municipio de Pará. Se observó que solo el $24 \%$ de los profesionales entrevistados se sometieron a reubicación sectorial, el $82 \%$ de los profesionales dijo que hubo cambios en el perfil de servicio de las unidades de salud, hubo necesidad de reprogramar la atención a los pacientes, el 88\% de los profesionales se sometió a rotación de actividades, $47 \%$ dijo que faltaban medicamentos para el tratamiento de la lepra en las unidades donde trabaja, 53\% dijo que no faltan materiales para el cuidado de los leprosos, hay dificultades en la programación de las pruebas de laboratorio, en la evaluación de contactos domiciliarios y sociales y en la derivación y contrarreferencia de pacientes. De estos datos se concluye que la pandemia Covid-19 generó dificultades para la atención y tratamiento de los pacientes con lepra en las unidades de atención primaria participantes, provocando retrasos en las citas, reprogramación de citas, extensiones de tratamiento por falta de medicación, dificultades en la evaluación de contactos y búsqueda activa de nuevos casos, contribuyendo a generar un impacto negativo en el programa y consecuentemente en el sistema de salud.

Palabras clave: Lepra; Covid-19; Pandemia; Profesionales de la salud.

\section{Introdução}

A hanseníase é uma doença infecciosa, crônica, causada pelo Mycobacterium leprae que acomete principalmente a pele e os nervos periféricos e que possui uma evolução lenta e crônica (Brasil, 2017). Apresenta um alto poder incapacitante, estigmatizante e com um passado histórico de discriminação e isolamento e está entre as causas básicas e associadas de óbitos no país, apesar da existência não mensurada de subnotificações, acometendo principalmente homens, analfabetos e idosos (Ramos et al., 2016).

O Brasil é um dos países que apresenta o maior número de casos novos da doença. Em 2016 a taxa de detecção na população geral foi de 12,23 sendo que desses, 0,84 foram detectados com grau II de deformidade. O percentual de cura na coorte no ano de 2017 ficou em 76,72\% (Brasil, 2018).

A análise dos indicadores em hanseníase permite retratar, dentro de um certo contexto, o reflexo da rede de assistência prestada pela rede de serviços através da medição de variáveis e comparação de parâmetros que se traduzem em um diagnóstico da situação analisada. Por exemplo, no ano de 2016 o estado do Mato Grosso apresentou uma taxa de detecção de 80,62, sendo que 3,05 foram com grau II de deformidade, apresentando um percentual de cura em 2017, para os anos da coorte, de 72,26\% (Brasil, 2018).

Os dados acima demonstram que a presença da doença e a força de transmissão recente da endemia ainda é alta no estado, com uma tendência oscilativa ao longo dos anos, estando seis vezes maior que a média nacional, sendo que em muitos casos o diagnóstico é feito de maneira tardia, gerando sequelas por vezes irreversíveis e que interferem diretamente na qualidade de vida do paciente. A taxa de cura está próxima da média nacional, porém vale ressaltar que em Mato Grosso a situação da hanseníase se configura em um sério problema de saúde pública demonstrando fragilidade na avaliação de contatos e diagnóstico precoce da doença, sendo também o abandono maior do que a média nacional.

Os coronavírus (CoVs) pertence a uma família de vírus de RNA zoonótico de fita simples da família coronavidae, causadores de síndromes respiratórias. O novo Coronavírus, denominado de SARS-CoV-2, causador da infecção COVID-19 foi detectado pela primeira vez na china na cidade de Wuhan no dia 31 de dezembro de 2019. É uma doença de rápida 
propagação, que se alastrou por diversos países do mundo causando inúmeros impactos e elevados índices de morbimortalidade jamais vista nos últimos séculos. A partir de 13 de maio de 2020 foram identificados mais de 4 milhões de casos da doença e mais de 283 óbitos relacionados a doença, declarada em janeiro do mesmo ano pela Organização Mundial da Saúde (OMS) como uma epidemia de emergência internacional e posteriormente, em março, como uma pandemia de grande repercussão (Zimmermann e Curtis, 2020) (Lana et al., 2020) (Xavier et al., 2020).

As manifestações clínicas do SARS-CoV-2 caracterizam-se principalmente por sintomas respiratórios e gastrointestinais, podem ser presentar por um resfriado comum a grave, bronquite, pneumonia, síndrome do desconforto respiratório agudo grave (SDRA), coagulopatia, falência de múltiplos orgãos, podendo causar morte. No entanto, a doença provocada por este vírus produz impactos diferentes quando observado a faixa etária, em crianças as manifestações da covid19, geralmente, apresentam-se na forma menos grave quando comparada a adultos (Zimmermann e Curtis, 2020).

A pandemia do Covid-19 fez com que vários municípios tivessem superlotações em seus serviços de saúde e que direcionasse serviços e equipes para o atendimento dos casos de infecção do novo Coronavírus.

A percepção da qualidade em saúde abrange diversas perspectivas entre elas a clínica e a populacional. A perspectiva clínica preocupa-se no impacto dos profissionais sobre a saúde dos usuários. A perspectiva populacional preocupa-se no impacto da saúde sobre a redução das disparidades na saúde dos subgrupos populacionais (Starfield, 2002).

O sistema de saúde brasileiro possui um grande desafio que é manter a qualidade dos serviços de hanseníase e assegurar que todas as pessoas afetadas pela doença, independentemente de onde morem, tenham iguais oportunidade de ser diagnosticadas e tratadas por profissionais da área de saúde competentes e treinados. Para que este desafio se traduza em ações e serviços, é necessário minimizar barreiras que dificultam o acesso da população aos serviços e garantir insumos, materiais e outras ferramentas necessárias ao desenvolvimento das ações do programa de hanseníase em todos os municípios brasileiros, tendo cada ente federativo, sua parcela de contribuição nesse processo (Opromolla \& Laurenti, 2011).

O próprio contexto histórico da hanseníase faz com que ela seja percebida pelos usuários como algo ruim, que gera tristeza, solidão, medo e vergonha. Os relatos de pacientes apontam para um histórico de discriminação social dentro do próprio contexto familiar e/ou do grupo de amigos, gerando quadros de desarmonia e instabilidade que resultam em sofrimento psíquico (Nunes et al., 2008).

Para Lanza, Vieira, Oliveira e Lana (2014), avaliar a hanseníase é de extrema importância por se tratar de um agravo prioritário na política de saúde do Brasil necessitando de ações que visem o fortalecimento da atuação da APS no seu controle e na qualidade dos serviços ofertados.

Percebe-se, através das buscas realizadas em base de dados e pelos estudos publicados que as pesquisas referentes à qualidade da assistência em saúde prestada à crianças e adolescentes com hanseníase ainda ocorrem de maneira tímida, sendo necessário maiores investimentos nesse tipo de estudo de modo a se estabelecer parâmetros para a melhoria da assistência prestada a essa clientela e consequentemente promover melhorias em sua qualidade de vida.

Tendo em vista o fator negligenciante da hanseníase enquanto doença nos serviços de saúde, este estudo teve como objetivo analisar como a pandemia do novo Coronavírus vem impactando o atendimento em hanseníase no município de Parauapebas, estado do Pará.

\section{Metodologia}

Trata-se de um estudo de avaliação de serviços, transversal, descritivo, baseado nos pressupostos teóricos e metodológicos de Donabedian $(1966 ; 1972 ; 1982)$ que trata da avaliação da qualidade em saúde, em uma dinâmica de acontecimentos e modelos complexos, estabelecendo que a saúde não pode ser vista como um evento isolado, uma vez que é 
constituída por processos de trabalho que integram avaliadores e avaliados na busca de melhorias para o serviço ou programa avaliado, visando uma maior eficiência e efetividade nas ações desenvolvidas.

O estudo foi realizado entre os meses de agosto a outubro de 2021, nas unidades de saúde da Atenção Primária em Saúde - APS da zona urbana do município de Parauapebas-Pa, mediante contato realizado com o gerente de cada estabelecimento assistencial. Parauapebas é um município brasileiro do estado do Pará, pertencente à mesorregião do Sudeste Paraense e sede da microrregião de Carajás. Localiza-se no norte brasileiro, distante 719 km da capital Belém (IBGE, 2021).

A população alvo do estudo foi composta por profissionais de saúde, médicos, enfermeiros e fisioterapeutas que atuam nas unidades de saúde que atendem casos de hanseníase. Os profissionais foram convidados a participar do estudo mediante visita realizada pelo pesquisador em cada unidade de saúde.

Fizeram parte do estudo médicos, enfermeiros e fisioterapeutas, atuantes em unidades de atenção primária, centros de referências ou outros locais que acompanham casos de pacientes com hanseníase a pelo menos 01 ano, independente do vínculo empregatício, selecionados por conveniência.

Foram excluídos deste estudo médicos, enfermeiros e fisioterapeutas atuantes em unidades hospitalares, internação ou que prestem apenas atendimento a casos de hanseníase sem o devido acompanhamento, que tenham sido remanejados a pouco tempo para a atenção primária em saúde ou que possuam menos de 01 ano de experiência no atendimento e acompanhamento de pacientes com hanseníase.

No total, foram distribuídos 46 instrumentos de coleta de dados, sendo 25 instrumentos para enfermeiros, 5 para fisioterapeutas e 16 para médicos, representando 10 unidades de APS. Deste total, participaram do estudo 07 (sete) enfermeiros, 03 (três) fisioterapeutas e 07 (sete) médicos atuantes em 07 (sete) unidades de APS.

A coleta de dados foi realizada através da entrega de um questionário estruturado, composto de 14 questões fechadas, com alternativas de respostas "Sim, Não e Não Sabe Informar/Não Lembra/Não se Aplica". O questionário utilizado foi elaborado especificamente para esse fim, não tendo sido realizado um processo metodológico de validação do instrumento, porém foi realizado uma pré-testagem do instrumento em uma amostra de 03 profissionais de saúde para avaliação da compreensão dos itens do questionário. O questionário foi entregue em 10 unidades de saúde da atenção primária do município.

As variáveis utilizadas dizem respeito às características sociodemográficas dos profissionais de saúde bem como a caracterização do perfil profissional e do serviço de saúde a qual está vinculado. Foram mapeados dados referentes ao atendimento realizado pelo profissional de saúde e a estrutura do serviço durante à pandemia atual.

Todos os participantes assinaram um Termo de Consentimento Livre e Esclarecido (TCLE) em 2 vias, ficando uma com o depoente e a outra em arquivo do pesquisador.

Para a análise estatística foi utilizado o programa Excel. Foram utilizadas análises descritivas, com uso de frequência absoluta e percentual, de modo a retratar com o máximo de fidedignidade possível o objetivo proposto. Os dados foram apresentados através de gráficos e quadros de modo a resumir e sintetizar as informações obtidas.

$\mathrm{O}$ presente estudo adotou todas as normas estabelecidas para pesquisa com seres humanos conforme preconiza a resolução do Conselho Nacional de Saúde - CNS nº 466/12, sendo submetido, apreciado e aprovado pelo Comitê de Ética em Pesquisa da Universidade do Estado do Pará, Campus XII-Tapajós, com parecer nº: 4.660.685, CAAE: 44032921.0.0000.5168.

\section{Resultados}

No instrumento de coleta de dados foram utilizadas várias variáveis que fossem capazes de mensurar o efeito da pandemia de Covid-19 no funcionamento do programa de hanseníase e no atendimento ao usuário do programa. 
A amostra do estudo foi composta por 17 profissionais de saúde atuantes nas unidades de saúde da APS. No total, foram distribuídos 46 instrumentos de coleta de dados, sendo 25 instrumentos para enfermeiros, 5 para fisioterapeutas e 16 para médicos, representando 10 unidades de APS. Do total de respondentes, $41 \%$ (7) são enfermeiros, 18\% (3) são fisioterapeutas e $41 \%$ (7) são médicos, atuantes em 7 unidades de saúde do total das unidades de APS que foram convidadas a participar do estudo, conforme demonstra o gráfico 1.

Gráfico 1: Caracterização da amostra de profissionais de saúde participantes do estudo - Parauapebas-Pa, Brasil, 2021.

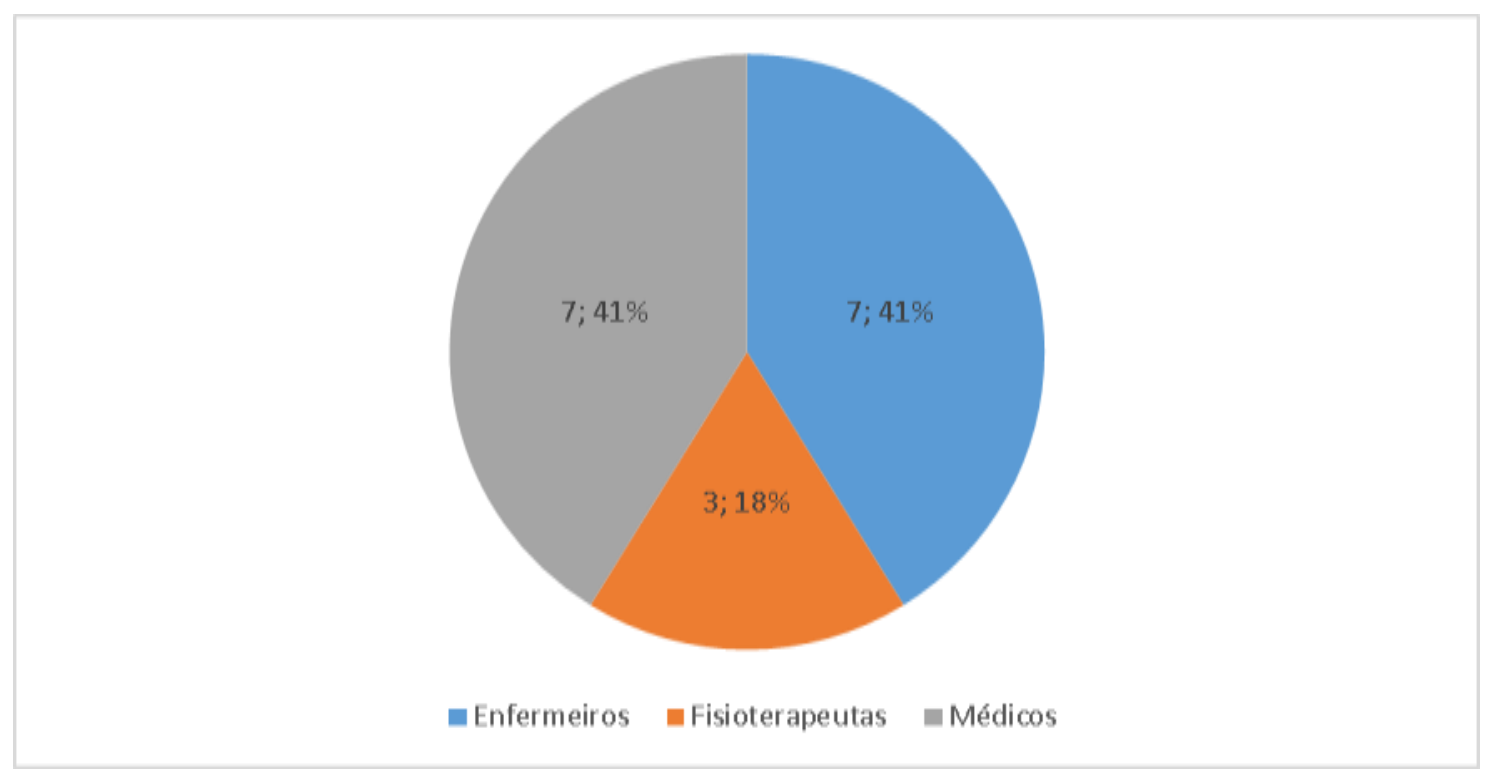

Fonte: Dados coletados pelos autores.

O Quadro 1 apresenta as demais variáveis do estudo, bem como sua divisão por categoria profissional e o total de respostas agregadas conforme detalhamento.

Quanto ao remanejamento de profissionais de saúde, das suas unidades de origem, durante a pandemia de Covid-19, o presente estudo demonstrou que, dentre os depoentes, apenas 04 (24\%) profissionais foram remanejados de setor ou de atividade nesse período de pandemia, sendo que 13 (76\%) dos profissionais continuaram exercendo suas atividades em suas unidades de saúde de origem desde o início da pandemia.

No que se refere a mudanças no perfil de atendimento das unidades de saúde da APS durante a pandemia de Covid19, os profissionais de saúde entrevistados em sua maioria relataram que houveram mudanças nesse período 14 (82\%). Observa-se que entre todos os grupos de profissionais, tanto enfermeiros, médicos e fisioterapeutas a maioria relataram terem passados por mudança no perfil de atendimento. Vale destaque para os fisioterapeutas em que $100 \%$ (3) dos entrevistados mudaram de perfil de atendimento

A necessidade de reagendamentos de atendimentos para os pacientes com hanseníase nas unidades de saúde da APS durante a pandemia de Covid-19, todos os grupos de profissionais relataram que por algum motivo os pacientes necessitaram reagendar os seus atendimentos, conforme detalhado por 4 (57\%) dos enfermeiros, 2 (67\%) dos fisioterapeutas e $3(43 \%)$ dos médicos.

Chama a atenção também a quantidade de depoentes que não souberam informar se houve ou não a necessidade de reagendamentos de atendimentos. Do total de respondentes, 5 (29\%) não souberam responder a esta questão.

Quanto ao rodízio de profissionais nas unidades de saúde da APS durante a pandemia de Covid-19, observa-se que a maioria dos profissionais afirmaram terem passado por esse tipo de modalidade de escala durante a pandemia, correspondendo a um total de 15 (88\%) depoentes. Os que afirmaram não terem passado por rodízio de profissionais somam 1 (6\%) e os que 
não souberam informar correspondem a $1(6 \%)$. Dentre os profissionais que mais passaram por rodízio foram os médicos onde $100 \%$ dos depoentes afirmaram terem passado por esse tipo de escala.

No que tange a falta de medicamentos para o tratamento da hanseníase nesse período, 47\% (8) da amostra responderam que em algum momento da pandemia por Covid-19 houve falta de medicamentos para os pacientes com hanseníase. Foi expressivo também o número de depoentes que responderam não saber desta informação, representando 6 $(35 \%)$.

No que se refere a falta de materiais para o cuidado e tratamento do paciente com hanseníase nesse período, a maioria dos depoentes 9 (53\%) afirmaram que não houve falta de materiais nesse período. Apenas 3 (18\%) depoentes afirmaram que em algum momento da pandemia houve falta de materiais para a hanseníase, enquanto 5 (29\%) afirmaram não saber de tal informação

Em relação à dificuldade para agendamento de exames laboratoriais necessários ao tratamento de hanseníase, a maioria dos enfermeiros 6 (86\%) afirmaram que os pacientes apresentaram algum grau de dificuldade nesse processo, enquanto que $4(57 \%)$ dos médicos afirmaram que não houve dificuldade para o agendamento. Em relação ao total de profissionais, 8 (47\%) afirmaram que existiram dificuldades para o agendamento durante a pandemia, 5 (29\%) afirmaram que não houveram dificuldades para o agendamento e 4 (24\%) não souberam informar.

Quando questionados se houve treinamentos sobre o manejo clínico da hanseníase no período de pandemia, 10 (59\%) dos profissionais, representando a maioria, afirmaram que não houveram treinamentos nesse período, enquanto que 5 (29\%) afirmaram terem passando por algum treinamento específico para a hanseníase nesse período, com destaque para os fisioterapeutas.

Quanto à realização de campanhas educativas para a prevenção e cuidado da hanseníase 8 (47\%) dos profissionais entrevistados não souberam responder a este questionamento. Em contrapartida, 6 (35\%) afirmaram que houve campanha educativa para a hanseníase.

Referente a avaliação de contatos domiciliares e sociais do paciente com hanseníase, 7 (41\%) dos entrevistados afirmaram que este tipo de avaliação ocorreu normalmente enquanto que $6(35 \%)$ dos profissionais afirmaram que a pandemia interferiu na avaliação desses contatos. Em relação a busca ativa de casos na comunidade, 5 (29\%) dos profissionais afirmaram que realizaram busca ativa, 7 (42\%) afirmaram que não realizaram busca ativa e 5 (29\%) não souberam informar sobre a ocorrência deste evento.

Quanto ao acompanhamento da dose supervisionada do tratamento para a hanseníase no período de pandemia, a maioria dos profissionais 12 (70\%) afirmaram que houve acompanhamento desse tipo de tratamento. No que tange aos procedimentos para referência e contrarreferência do paciente com hanseníase para outros serviços, 11 (69\%) dos profissionais afirmaram que encontraram alguma dificuldade nesse tipo de manejo do paciente. 
Quadro 1: Distribuição das respostas dos profissionais de saúde em relação às variáveis de estudo - Parauapebas-Pa, Brasil, 2021.

\begin{tabular}{|c|c|c|c|c|c|c|c|c|c|c|c|c|}
\hline \multirow[t]{2}{*}{ VARIÁVEL } & \multicolumn{3}{|c|}{ ENFERMEIROS } & \multicolumn{3}{|c|}{$\begin{array}{l}\text { FISIOTERAPEU } \\
\text { TAS }\end{array}$} & \multicolumn{3}{|c|}{ MÉDICOS } & \multicolumn{3}{|c|}{ TOTAL } \\
\hline & $\mathrm{S}$ & $\mathrm{N}$ & NSI & $\mathrm{S}$ & $\mathrm{N}$ & NSI & $\mathrm{S}$ & $\mathrm{N}$ & NSI & $\mathrm{S}$ & $\mathrm{N}$ & NSI \\
\hline $\begin{array}{l}\text { Necessidade de remanejamento de profissionais de } \\
\text { setor ou função }\end{array}$ & 1 & 6 & - & 1 & 2 & - & 2 & 5 & - & 4 & 12 & - \\
\hline $\begin{array}{l}\text { Houve mudança no perfil de atendimento na } \\
\text { unidade de Atenção Primária }\end{array}$ & 5 & 1 & 1 & 3 & - & - & 6 & 1 & - & 14 & 2 & 1 \\
\hline $\begin{array}{l}\text { Houve necessidade de reagendamentos de } \\
\text { atendimentos para os pacientes com hanseníase na } \\
\text { unidade de Atenção Primária }\end{array}$ & 4 & 1 & 2 & 2 & - & 1 & 3 & 2 & 2 & 9 & 3 & 5 \\
\hline $\begin{array}{l}\text { Houve rodízio de profissionais na Unidade de } \\
\text { Saúde }\end{array}$ & 6 & - & 1 & 2 & 1 & - & 7 & - & - & 15 & 1 & 1 \\
\hline $\begin{array}{l}\text { Faltou medicamentos para o tratamento da } \\
\text { hanseníase }\end{array}$ & 4 & 1 & 2 & 1 & - & 2 & 3 & 2 & 2 & 8 & 3 & 6 \\
\hline $\begin{array}{l}\text { Faltou materiais para o cuidado e tratamento da } \\
\text { hanseníase }\end{array}$ & 3 & 2 & 2 & - & 2 & 1 & - & 5 & 2 & 3 & 9 & 5 \\
\hline $\begin{array}{l}\text { Houve dificuldades no agendamento de exames } \\
\text { laboratoriais }\end{array}$ & 6 & - & 1 & - & 1 & 2 & 2 & 4 & 1 & 8 & 5 & 4 \\
\hline $\begin{array}{l}\text { Houve treinamentos sobre o manejo clínico da } \\
\text { hanseníase }\end{array}$ & 2 & 5 & - & 2 & - & 1 & 1 & 5 & 1 & 5 & 10 & 2 \\
\hline $\begin{array}{l}\text { Houve realização de campanhas educativas sobre a } \\
\text { hanseníase }\end{array}$ & 1 & 1 & 5 & 1 & - & 2 & 4 & 2 & 1 & 6 & 3 & 8 \\
\hline Houve avaliação de contatos domiciliares e sociais & 3 & 3 & 1 & 1 & 0 & 2 & 3 & 3 & 1 & 7 & 6 & 4 \\
\hline Busca ativa de casos na comunidade & 1 & 5 & 1 & 2 & - & 1 & 2 & 2 & 3 & 5 & 7 & 5 \\
\hline Acompanhamento da dose supervisionada & 6 & 1 & - & 3 & - & - & 3 & - & 4 & 12 & 1 & 4 \\
\hline $\begin{array}{l}\text { Dificuldades na referência/contrarreferência do } \\
\text { paciente }\end{array}$ & 6 & 1 & - & 2 & - & 1 & 3 & 1 & 3 & 11 & 2 & 4 \\
\hline
\end{tabular}

S: sim; N não; NSI não soube informar Fonte: Dados obtidos pelos autores.

\section{Discussão}

Quando se compara a quantidade de instrumentos de pesquisas distribuídos por grupo de profissionais, observa-se que houve uma grande adesão dos profissionais fisioterapeutas, seguidos pelos médicos.

Embora as maiores quantidades de questionários tenham sido distribuídas para os enfermeiros, estes, proporcionalmente, apresentaram o menor índice de resposta. Isto pode se justificar pelas inúmeras atividades desenvolvidas por estes profissionais que possuem uma sobrecarga de serviço (Caçador et al., 2015).

No presente estudo observou-se um baixo índice de remanejamento de profissionais de saúde nas unidades pesquisadas embora estudos recentes e as principais manchetes do país relatem que nesse período pandêmico foi comum o remanejamento de médicos, enfermeiros e fisioterapeutas para outras funções (Beraldo, 2020) (Clementino et al, 2020).

Observou-se neste período de pandemia que uma grande parte dos serviços de saúde, tanto na atenção primária em saúde quanto a nível hospitalar, passaram por mudanças no perfil de atendimento e reestruturação dos serviços de modo a contemplar além das atividades bases inerentes, também os pacientes acometidos por Covid-19 e suas complicações (Bergallo, 2020) (Jardim et al, 2021).

O cancelamento de consultas durante a pandemia e sua consequente necessidade de reagendamentos dos atendimentos previstos geram grandes transtornos no seguimento e acompanhamento do paciente com doenças crônicas ou de longo tratamento como a hanseníase. Esse fato pode acarretar complicações como o agravamento do estado de saúde do paciente e o desenvolvimento de reações e sequelas (Santos Silva, 2021).

Referente ao rodízio de profissionais, a pandemia de Covid-19 criou um cenário de trabalho remoto, definições de atividades essenciais e etc. Em relação aos profissionais de saúde o que se observou foi um aumento da sobrecarga de trabalho com reduções dos períodos de descansos, aumento da carga horária de atividades e aumento na demanda de atendimentos 
(Heliotério et al., 2020). Esses dados corroboram os achados neste estudo uma vez que a maioria dos depoentes afirmaram não terem realizado rodízio de atividades ou de serviço.

Neste estudo, uma grande parte dos profissionais vivenciou a falta de medicamentos para o tratamento da hanseníase nas unidades de saúde da atenção primária neste período de pandemia. Foi frequente as manchetes de jornais e notícias informando que o Brasil estava passando por uma falta de medicamentos para hanseníase nesse período. A falta de medicamento acarreta vários outros problemas como a priorização de pacientes, o retrocesso no controle da doença, o aumento na transmissão e a dificuldade no diagnóstico (Neves, 2021).

Em compensação, a maioria dos participantes do estudo afirmaram não terem presenciado a falta de materiais para o cuidado e tratamento dos pacientes com hanseníase. Nesses materiais incluem-se soro fisiológico, hidratantes a base de ureia, colírio para reposição de lágrimas, protetor solar entre outros. É coerente se pensar que esses materiais não faltaram com tanta frequência como a medicação por serem adquiridos pelo próprio município e não dependerem de envio pelo governo federal via Ministério da Saúde.

A sobrecarga de exames para o diagnóstico e a sequência de tratamento de Covid-19 gerou um efeito cascata nos exames de rotina para várias doenças tratadas a nível de ambulatório e unidades de saúde. Com isso, houve uma dificuldade no agendamento de exames para a hanseníase, o diabetes, a hipertensão arterial e outras doenças uma vez que a prioridade nesse período foram os exames para o tratamento e diagnóstico de Covid-19. Aumentou-se a demanda, porém a maioria das estruturas físicas, materiais e de recursos humanos não foram providas de aumento em seu dimensionamento (SBOC, 2020).

Além de todos esses prejuízos, a pandemia trouxe consigo uma redução no número de treinamentos para manejo da hanseníase e nas realizações de campanhas educativas. Isso acarreta uma queda nas taxas de diagnóstico e um aumento no estigma da doença uma vez que existe um desvio de foco onde se prioriza o Covid-19 em detrimento de outras doenças que também assolam o país (Siqueira, 2021).

A priorização da pandemia também reduziu de certa forma a avaliação de contatos domiciliares e sociais e a busca ativa de casos novos na comunidade uma vez que muitos serviços mudaram o perfil de atendimento nesse período. Isso, juntamente com a redução das campanhas e atividades educativas contribuem para um futuro aumento nas taxas de detecção e transmissibilidade da doença (Bergallo, 2020) (Jardim et al, 2021) (Siqueira, 2021).

Apesar de tantos prejuízos, o estudo demonstrou que o acompanhamento da dose supervisionada no tratamento da hanseníase continua sendo feito sem alterações na maioria das unidades de saúde, desde que exista a disponibilidade do medicamento. A dose supervisionada possui uma grande importância no tratamento da hanseníase e é considerada um fator de qualidade na avaliação do programa (Mantellini et al., 2019).

As dificuldades encontradas para a referência e contrarreferência dos pacientes com hanseníase para outros serviços se justifica também pela mudança de perfil do atendimento das unidades de saúde. Com isso, o acesso a serviços de referência ficou comprometido devido ao atendimento para Covid-19, necessidade de remanejamento de profissionais, trabalho remoto e outras especificidades trazidas pela pandemia (Bergallo, 2020) (Jardim et al, 2021).

Este estudo possui como limitação o fato da amostra ter sido pequena, embora tenha conseguido alcançar um número representativo de unidades de saúde. É necessário que sejam realizados novos estudos, com uma amostragem maior e que envolvam também outros municípios, em formato multicêntrico, permitindo assim um melhor retrato desta situação analisada

\section{Conclusão}

A partir deste estudo observou-se que a pandemia de Covid-19 criou dificuldades para o atendimento e tratamento do paciente com hanseníase nas unidades de saúde da atenção primária em saúde em Parauapebas, gerando atrasos em agendamentos, remarcações de consultas, prorrogações de tratamento devido à falta de medicação, dificuldades na avaliação 
de contatos e busca ativa de novos casos, contribuindo para gerar um impacto negativo no programa e consequentemente no sistema de saúde.

A redução nos treinamentos e campanhas educativas contribuem para gerar um sentimento de insegurança nos profissionais de saúde que lidam com a hanseníase no dia a dia. Assim, o impacto do Covid-19 na atenção ao paciente com hanseníase não será apenas um problema pontual e passageiro uma vez que ainda será possível sentir os efeitos dessas consequências a longo prazo.

Recomenda-se a realização de novos estudos que contemplem comparações com dados epidemiológicos da doença no período de pandemia, de modo a retratar o real impacto da Covid-19 nas estatísticas da hanseníase. Sugere-se também a realização de estudos de abordagem mista, que visem contemplar as lacunas de compreensão da percepção de profissionais e usuários, em relação aos efeitos da pandemia de Covid-19 no atendimento ao paciente com hanseníase.

\section{Referências}

Beraldo, R. (2020). Especial Covid-19: trabalhadores da saúde em pandemias: 1918 e 2020. Casa de Osvaldo Cruz. http://coc.fiocruz.br/index.php/pt/todasas-noticias/1821-trabalhadores-da-saude-em-pandemias-1918-e-2020.html

Bergallo, R. (2020). Covid-19: como organizar a UBS para manter o cuidado integral durante a pandemia? PEBMED. https://pebmed.com.br/covid-19como-organizar-a-ubs-para-manter-o-cuidado-integral-durante-a-pandemia/\#: :text=As\%20visitas\%20domiciliares $\% 20 \mathrm{devem} \% 20$ ser,internet $\% 20$ (aplicati vos\%20como $\% 20$ Whatsapp).

Brasil. Ministério da Saúde. Secretaria de Vigilância em Saúde. Departamento de Vigilância das Doenças Transmissíveis. Guia Prático sobre a Hanseníase. (2020). Brasília: Ministério da Saúde. http://portalarquivos2.saude.gov.br/images/pdf/2017/novembro/22/Guia-Pratico-de-Hanseniase-WEB.pdf.

Brasil. Ministério da Saúde. Sala de Apoio à Gestão Estratégica. Situação de Saúde. Indicadores de Morbidade. Hanseníase. Brasil. Mato Grosso. (2019). Brasília: Ministério da Saúde. http://sage.saude.gov.br/\#.

Brasil. Ministério da Saúde. Secretaria de Atenção em Saúde. Departamento de Atenção Básica. Informe da atenção básica $n^{o} 42$. (2007). Brasília: Ministério da Saúde. http://bvsms.saude.gov.br/bvs/publicacoes/07_1120_P.pdf

Caçador, B. S., Brito, M. J. M., Moreira, D. A., Rezende, L. C. \& Vilela, G. S. (2015). Ser enfermeiro na estratégia de saúde da família: desafios e possibilidades. Rev Min Enferm., 19(3), 620-626. http://reme.org.br/artigo/detalhes/1027.

Clementino, F. S., Chaves, A. E. P. Pessoa Júnior, J. M., Miranda, F.A N., Medeiros, S. N. \& Martiniano, C. S. (2020). Nursing care provided to people with covid-19: challenges in the performance of the cofen/corens system. Texto \& Contexto - Enfermagem [online]., 29(e20200251). https://doi.org/10.1590/1980265X-TCE-2020-0251.

Donabedian, A., Wheeler, H. R. C. \& Wysze-Wianski, L. (1982). Quality, Cost, and Health: An Integrative Model. Med. Care 20(10), 1975-92.

Donabedian, A. (1966). Evaluating the Quality of Medical Care. Milbank Mem. Fund. Q. 44,166, Part 2.

Donabedian, A. (1978). The Quality of Medical Care. Science 200.

Jardim, T. V., Jardim, F. V., Jardim, L., Coragem, J. T., Castro, C. F., Firmino, G. M., \& Jardim, P. (2021). Changes in the Profile of Emergency Room Patients during the COVID-19 Outbreak in a General Hospital Specialized in Cardiovascular Care in Brazi. Arquivos brasileiros de cardiologia, 116(1), 140143. https://doi.org/10.36660/abc.20200595

Lana, R. M., Coelho, F. C., Gomes, M. F. C., Cruz, O. G., Bastos, L. S., Villela, D.A. M. \& Codeço, C. T. (2020). Emergência do novo coronavírus (SARSCoV-2) e o papel de uma vigilância nacional em saúde oportuna e efetiva. Cad. Saúde Pública, 36(3), e00019620 https://doi.org/10.1590/0102$311 \times 00019620$

Lanza F. M., Vieira, N. F., Oliveira, M. M. C. \& Lana, F. C. F. (2014). Instrumento para avaliação das ações de controle da hanseníase na Atenção Primaria. Rev Bras Enferm., 67(3), 339-46. http://www.scielo.br/pdf/reben/v67n3/0034-7167-reben-67-03-0339.pdf.

Mantellini, G. G., Gonçalves, A. \& Padovani, C. R. (2019). Políticas públicas referentes às incapacidades físicas em hanseníase na virada do século: uma década de (des)controle? Physis: Revista de Saúde Coletiva [online]., 29(1). https://doi.org/10.1590/S0103-73312019290105.

Neves, U. (2021). Hanseníase: Brasil enfrenta desabastecimento de medicamentos. PEBMED. https://pebmed.com.br/hanseniase-brasil-enfrentadesabastecimento-de-

medicamentos/\#: :text=O\%20Brasil\%20enfrenta\%20um\%20desabastecimento,chamados\%20de\%20poliquimioterapia\%2C\%20ou\%20PQT.

Nunes, J. M.., Oliveira, E. M. \& Vieira, N. F. C. (2008). Ter hanseníase: percepções de pessoas em tratamento. Rev Rene., 9(4), 99-106. http://www.redalyc.org:9081/html/3240/324027964012/

Oncoguia (2020. SBOC alerta sobre riscos no adiamento de exames preventivos. Oncoguia. http://www.oncoguia.org.br/conteudo/sboc-alerta-sobre-riscosno-adiamento-de-exames-preventivos-durante-a-quarentena/13668/7/ 
Research, Society and Development, v. 11, n. 2, e4111225459, 2022

(CC BY 4.0) | ISSN 2525-3409 | DOI: http://dx.doi.org/10.33448/rsd-v11i2.25459

Opromolla, P. A.\& Laurenti, R. (2011). Hansen's disease control in the State of São Paulo: a historical analysis. Rev. Saúde Pública, 45(1), 195-203. http://www.scielo.br/scielo.php?script=sci_arttext\&pid=S0034-89102011000100022\&lng=en\&nrm=iso

Ramos, A. R. S., Ferreira, S. M. B.\& Ignotti, E. (2016). Óbitos atribuídos à hanseníase no Brasil (2000 a 2007). In: Brunken, G. S., Muraro, A. P.\& Scatena, J. $\mathrm{H}$ (Coords.). Estudos de morbidade e cronicidade. EdUFMT. p. 100-18.

Starfield, B. (2004). Atenção Primária: equilíbrio entre necessidades de saúde, serviços e tecnologia. (2a ed.), Ministério da Saúde/Unesco/DFID.

Santos Silva, J.M., Nascimento, D. C., Moura, J.C.V., Almeida, V. R. S., Freitas, M. Y. G. S., Santos, S. D., Melo, A. M. S., Silva, D. A. C., Dias, J. S. \& Silva, I. R. S. (2021). Atenção às pessoas com hanseníase frente à pandemia de Covid-10: uma revisão narrativa. Revista Eletrônica Acervo Saúde.,. 13(2). https://acervomais.com.br/index.php/saude/article/view/6124.

Siqueira, R. (2021). Pandemia provoca queda no diagnóstico de hanseníase e preocupa autoridades. Jornal da USP. https://jornal.usp.br/atualidades/pandemia-provoca-queda-no-diagnostico-de-hanseniase-e-preocupa-autoridades/\#: :text=Pandemia\%20provoca\%20

queda $\% 20 \mathrm{n} \quad \mathrm{o} \% 20 \mathrm{diagn} \% \mathrm{C} 3 \% \mathrm{~B} 3 \mathrm{stico} \% 20 \mathrm{de} \% 20$ hansen $\% \mathrm{C} 3 \%$ ADase $\% 20 \mathrm{e} \% 20$ preocupa $\% 20$ autoridades,-Marco\%20Andrey $\% 20 \mathrm{Cipriani} \&$ text=O $\% 20$ Brasil\%20registrou\%20queda\%20no,14\%20mil\%20no\%20ano\%20passado.

Xavier, A. R., Silva, J. S., Almeida, J. P. C. L., Conceição, J. F. F., Lacerda, G. S. \& Kanaan, S. (2020). COVID-19: manifestaç̃es clínicas e laboratoriais na infecção pelo novo Coronavírus. J. Bras. Patol. Med. Lab., 56 (e3232020) https://doi.org/10.5935/1676-2444.20200049.

Zimmermann, P., \& Curtis, N. (2020). Coronavirus Infections in Children Including COVID-19: An Overview of the Epidemiology, Clinical Features, Diagnosis, Treatment and Prevention Options in Children. The Pediatric infectious disease journal,39(5), 355-368. https://doi.org/10.1097/INF.0000000000002660 